\title{
Behaviour desorption study of the essential oil of Cedrus atlantica in a porous clay versus insecticidal activity against Sitophilus granarius: explanation of the phenomenon by statistical studies
}

\author{
Ayoub Ainane ${ }^{1}$, Fatouma Mohamed Abdoul-Latif ${ }^{2}$, Jalludin Mohamed ${ }^{2}$, Wissal Attahar ${ }^{1}$, Manar Ouassil ${ }^{1}$, \\ Zine Laabidine Shybat ${ }^{1}$, Adnane El Yaacoubi ${ }^{1}$, and Tarik Ainane ${ }^{1, *}$ \\ ${ }^{1}$ Superior School of Technology, University of Sultan Moulay Slimane, PB 170, Khenifra 54000, Morocco \\ ${ }^{2}$ Medicinal Research Institute, Center for Research and Study of Djibouti, BP 486, Djibouti
}

Received: 14 February 2021 / Accepted: 15 May 2021

\begin{abstract}
Substances of natural origin and more particularly essential oils currently represent an alternative solution in the fight for the protection of stored foodstuffs. The way to use these essential oils and the storage conditions remain the main handicap in dealing with this subject. This article develops the use of porous supports as a medium for the study of the behavior of essential oils with respect to insecticidal activities. The process relates to the fixing and/or grafting of essential oils in porous clay media in a well-defined geometric form included in the storage methods. The study of transfers of the essential oil of Cedrus atlantica in a porous clay medium in the case of desorption was made by analytical and numerical models of diffusion process, to know the behavior of the oil and to determine some physical parameters (diffusivity $\mathrm{D}_{\mathrm{z}}$, activation energy $\mathrm{E}_{\mathrm{a}}$, evaporation rate $\mathrm{F}$ and constant of evaporation $\mathrm{K}$ ) which explain the mechanisms involved and to try to exploit them in parallel with the insecticidal activities against of Sitophilus granarius the main cereal pest by statistical approaches such as: design of experiments and principal component analysis.
\end{abstract}

Keywords: Clay / essential oil / behaviour desorption / diffusion / insecticidal activity

\section{Introduction}

Cereal storage is designed to keep grains for later use from many external factors, such as humidity, temperature, and light, that can lead to their degradation [1]. In addition, good storage also protects its content from other influences such as: microorganisms, shocks, odours, vibrations, dust and compressive forces [2]. The Technological development has brought the possibility to access to countless foods from every part of the globe, so the importance of protecting their organoleptic properties is food quality conformity [3-5].

More precisely, stored cereals are prone to postharvest loss in quality and quantity due to infestation by different groups of insects, which is considered the principal problem of storage [6]. Among the stored product beetles, Sitophilus granaries (Coleoptera: Dryophthorinae) are a primary pest in storage of grain-based products, principally wheat, but also attack others cereals: oats, rye, barley, corn, as well as derived products (flour) and other seeds, especially

\footnotetext{
* Corresponding author: t. ainane@usms.ma
}

Fabaceae $[7,8]$. Control of these insects relies heavily on the treatment by synthetic insecticides such as pyrethroids, organochlorines, organophosphates, carbamates and fumigants (mainly methyl bromide and phosphine) [9]. However, due to toxicity to consumers, insecticide resistance and resurgence of pests associated with synthetic insecticides, alternative solutions are requiring sought [10]. Pesticides from natural sources, relatively cheaper, biodegradable, which are means accessible and available, less toxic to non-target organisms and less prone to resistance by insect species are considered potential resources [11]. Among these resources, Essential oils produced from aromatic and medicinal plants have received much work due to their broad spectrum of activities, they are volatile, complex compounds characterized by a strong odour and are formed by aromatic plants as secondary metabolites (monoterpenes, sesquiterpenes and phenylpropanoid compounds) which show different insecticide properties (larvicidal, antifeedant, repellent, fumigant and ovicidal activities) [12]. Due forward described properties, essential oils are potentially suitable for integrated pest management programmes [13]. 
Table 1. Chemical composition (mass \%) of the clay.

\begin{tabular}{lllllllllll}
\hline $\mathrm{SiO}_{2}$ & $\mathrm{Al}_{2} \mathrm{O}_{3}$ & $\mathrm{Fe}_{2} \mathrm{O} 3$ & $\mathrm{~K}_{2} \mathrm{O}$ & $\mathrm{Na}_{2} \mathrm{O}$ & $\mathrm{MgO}$ & $\mathrm{TiO}_{2}$ & $\mathrm{CaO}$ & $\mathrm{P}_{2} \mathrm{O}_{5}$ & $\mathrm{SO}_{3}$ & $\mathrm{LOI}^{*}$ \\
\hline 33.40 & 17.03 & 32.47 & 3.27 & 3.60 & 1.24 & 0.69 & 0.68 & 0.10 & 0.01 & 8.35 \\
\hline
\end{tabular}

${ }^{*}$ LOI: loss of ignition at $1200^{\circ} \mathrm{C}$.

Cedar extracts considered an effective natural pesticide, especially their essential oil. This last obtained from different geographically regions, such as Morocco, Algeria, Cyprus, Lebanon, Syria, Turkey, Afghanistan and Himalayas, have been reported with their use in entomology to obtain many treatments traditionally ascribed to insecticidal activities [14]. In terms of previous reports on the chemical composition of this plant, the oil from the wood of this species has been extensively studied, sesquiterpenoids of the himachalane compounds being the major components [15].

The objective of this work is to carry out a desorption transfer study of the essential oil of Cedrus atlantica after fixation in a porous clay, by the use of experimental designs (complete factorial design) and analytical models of diffusion process (2nd Fick's Law), to know the process of essential oil and to determine the relation of certain physical parameters between them (diffusivity, constant of evaporation and rate of evaporation) which can explain the mechanism in play with the insecticidal activity against Sitophilus granaries.

\section{Materials and methods}

\subsection{Materials}

\subsubsection{Clay}

The studied natural porous clay RC was selected from Bejaad, Morocco (32 47'01.7'N 6²13'52.3'W). The chemical composition of the clay raw material (Tab. 1) shows that the major elements are $\mathrm{SiO}_{2}, \mathrm{Fe}_{2} \mathrm{O}_{3}$ and $\mathrm{Al}_{2} \mathrm{O}_{3}$. The amounts of $\mathrm{K}_{2} \mathrm{O}, \mathrm{Na}_{2} \mathrm{O}$ and $\mathrm{MgO}$ are significant. From $\mathrm{XRD}$ analyses (Fig. 1), it is found that this clay is mainly constituted of quartz, calcite, dolomite, kaolinite, illite and hematite [16].

\subsubsection{Essential oil of cedrus atlantica}

The essential oil of Cedrus atlantica (EOCa), which was obtained by steam distillation from the sawdust of Cedrus atlantica Man. from Morocco, was analysed by chromatography-mass spectrometry (GC-MS). The GC analysis was performed on SHIMADZU GC-14B equipped with and FID detector and a LM-5 $(30 \mathrm{~m} \times 0.25 \mathrm{~mm} \times 0.3 \mathrm{~mm})$ capillary column. The components in the oil were identified by comparison of the mass spectra obtained by CG-MS and literature data. The results obtained by the analysis are demonstrated in Table 2. In total were identified 22 constituents $(92.10 \%)$.

The data obtained with the chemical analysis are in accord with previous works reported in literature of the compounds analysed, the sesquiterpenes hydrocarbons $\alpha$-himachalene (15.63\%), $\beta$-himachalene $(31.24 \%)$ and

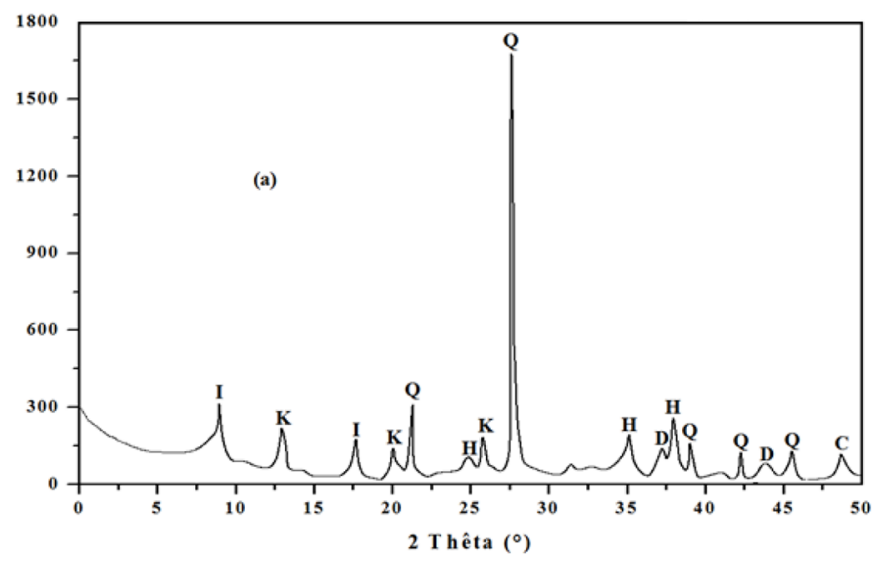

Fig. 1. XRD diffractograms of the clay RC.

Table 2. Chemical constitution of the EOCa.

\begin{tabular}{lcc}
\hline Components & $\begin{array}{l}\mathrm{RT} \\
(\mathrm{min})\end{array}$ & $\begin{array}{l}\text { Concentration } \\
(\%)\end{array}$ \\
\hline Limona ketone & 3.17 & 0.51 \\
$\alpha$-Longipinene & 6.88 & 0.16 \\
1,3-Dimethylindane & 7.64 & 0.31 \\
Aromandendrene & 7.75 & 1.52 \\
Isoledene & 7.86 & 1.19 \\
$\alpha$-Himachalene & 8.73 & 15.63 \\
$\boldsymbol{\gamma}$-Himachalene & 9.22 & 14.46 \\
$\beta$-Himachalene & 9.88 & 31.24 \\
$\alpha$-Dehydro-ar-himachalene & 9.95 & 2.06 \\
$\boldsymbol{\gamma}$-Dehydro-ar-himachalene & 10.07 & 3.67 \\
$\delta$-Cadinene & 10.13 & 1.93 \\
$\alpha$-Calacorene & 10.27 & 1.25 \\
$\alpha$-Bisabolene & 10.47 & 1.08 \\
Himachalene oxide & 10.70 & 1.21 \\
(+)- $\beta$-Himachalene oxide & 11.20 & 2.3 \\
Di-epi-1,10-cubenol & 11.51 & 1.42 \\
$\delta$-Cadinol & 11.66 & 1.09 \\
Allo-Himachalol & 11.89 & 1.62 \\
Deodarone & 12.54 & 2.73 \\
(E)- $\boldsymbol{\gamma}$-Atlantone & 12.78 & 1.2 \\
(Z)- $\alpha$-Atlantone & 13.00 & 1.22 \\
(E)-Atlantone & 13.82 & 4.3 \\
\hline
\end{tabular}

$\gamma$-himachalene (14.46\%) are the majority compounds, in which the component $\beta$-himachalene is the most abundant and represent practically the two-thirds of a percentage of the oil composition [17]. 


\subsubsection{Preparation of the mixture clay/essential oil}

The RC clay was homogenized, finely ground $(<63 \mu \mathrm{m})$ and heated for $2 \mathrm{~h}$ at $200^{\circ} \mathrm{C}$ for the organic complete combustion. a well-defined volume of EOCa has been mixed with a quantity of clay powder, prepared between 10 and $40 \%(\mathrm{v} / \mathrm{w})$ in order to have a total fixation of the oil on the powder. Then, the prepared materials $(\mathrm{RC}+\mathrm{EOCa})$ are moved to metal cylinders (diameters $1 \mathrm{~cm}$ and $2 \mathrm{~cm}$ ), for the continuation of the experimental works.

\subsection{Theoretical considerations}

\subsubsection{Assumptions}

- Material transfer of EOCa is considered with onedimensional diffusion in the cylindrical coordinate system.

- The diffusion takes place under transient conditions with a constant diffusivity and the rate of evaporation.

- During sorption, the concentration of EOCa on clay RC face reaches the equilibrium value as soon as the EOCa is mixed with RC.

- At the beginning of desorption, the concentration of EOCa throughout the clay is constant.

\subsubsection{Model of transfer of matter by diffusion}

Fick's second law for diffusion in terms of cylindrical coordinates $(\mathrm{r}, \theta, \mathrm{z})$, can be written as $[18,19]$ :

$$
\frac{\partial C}{\partial t}=\frac{1}{r}\left\{\frac{\partial}{\partial r}\left(r D_{r} \frac{\partial C}{\partial r}\right)+\frac{\partial}{\partial \theta}\left(\frac{D_{\theta}}{r} \frac{\partial C}{\partial \theta}\right)+\frac{\partial}{\partial z}\left(r D_{z} \frac{\partial C}{\partial z}\right)\right\} .
$$

Since, the cylinder height is smaller in front of the diameter and the lateral cylinder surface, the diffusion transfer will be directed in the direction of the height, which allows to consider the transfer model is onedimensional. The problem is made easier and the diffusion equation becomes Fick's 2nd Law:

$$
\frac{\partial C}{\partial t}=D_{z} \frac{\partial^{2} C}{\partial z^{2}}
$$

Considering the following boundary conditions:

$$
\left\{\begin{array}{lll}
t=0 & C=C_{0} & 0 \leq z \leq h \\
t>0 & C=C_{t} & z=h .
\end{array}\right.
$$

The analytical solution of equation (2) has been demonstrated by Crank (1979) [18] according to the conditions displayed in equation (3), it can be written as follows:

$C(z, t)=\frac{4}{\pi} \sum_{n=0}^{\infty} \exp \left(-D_{z} \cdot \frac{(2 n+1)^{2}}{h^{2}} \cdot \pi^{2} \cdot t\right) \cdot \cos \left(\frac{(2 n+1) \pi}{2 h} \cdot z\right)$.

The total mass $\mathrm{M}_{\mathrm{t}}$ of EOCa in the clay $\mathrm{RC}$ at an instant $\mathrm{t}$ is obtained by integration of the variable $\mathrm{C}$ (concentration) on the thickness of the material, and on a surface $\mathrm{S}$ subjected to the concentration flow:

$$
M_{t}=\int_{0}^{h} S . C(z, t) d z .
$$

The analytical solution of these equations taking into account the boundary conditions and after an infinite desorption time tends towards equilibrium is given by:

$$
\frac{M_{t}-M_{\infty}}{M_{0}-M_{\infty}}=\frac{8}{\pi^{2}} \sum_{n=0}^{\infty} \frac{1}{(2 n+1)^{2}} \exp \left(-D_{z} \cdot \frac{(2 n+1)^{2}}{h^{2}} \cdot \pi^{2} \cdot t\right)
$$

with:

$\mathrm{M}_{0}$ : The initial mass of EOCa absorbed.

$\mathrm{M}_{\mathrm{t}}$ : The mass of EOCa desorbed after a given time of aging.

$\mathrm{M}_{\infty}$ : the mass of liquid at equilibrium.

The simplifying the equation (6) by taking the first term of the series solution and assuming $\mathrm{M}_{\infty}=0$ :

$$
\frac{M_{t}}{M_{0}}=\frac{8}{\pi^{2}} \exp \left(\frac{-D_{z} \cdot \pi^{2} \cdot t}{h^{2}}\right)
$$

The term $\left(8 / \pi^{2}\right)$ is assumed to be equal to 1 . The last equation becomes:

$$
\frac{M_{t}}{M_{0}}=\exp \left(\frac{-D_{z} \cdot \pi^{2} \cdot t}{h^{2}}\right)
$$

During our study of the modelling and in consideration of the theoretical approximations already cited, we will focus on the three parameters which explain the desorption process: Diffusivity $\mathrm{D}_{\mathrm{z}}$, Evaporation constant $\mathrm{K}$ and evaporation rate $\mathrm{F}$ :

- The diffusivity does practically obtaining by the linear, logarithmic extrapolation of equation (8).

- the constant of evaporation $\mathrm{K}$ approximated to the diffusivity by the following equation:

$$
K=\frac{\pi^{2} \cdot D_{z}}{h^{2}} .
$$

- The evaporation rate $\mathrm{F}$ was determined using the initial value of the desorption flux, it is the value of the initial gradient of the desorption curve as a function of time, as indicated in the equation (10):

$$
F=F_{0}=\frac{1}{S} \cdot \lim _{t \rightarrow 0} \frac{d M}{\mathrm{~d} t} .
$$

\subsection{Experimentation and modelling}

\subsubsection{Insecticidal activity test}

An insecticide-susceptible population of the adult weevil species (Sitophilus granarius) were used in this study. The population of Sitophilus granarius was obtained from the Department of environmental engineering of EST-Khenifra (University of Sultan Moulay Slimane, Khenifra, Morocco). The strain was reared on wheat grains free of insecticide residue in glass containers $(1 \mathrm{~L})$ within growth chambers at $27 \pm 2{ }^{\circ} \mathrm{C}, 70 \pm 10 \%$ of relative humidity $(\mathrm{RH})$, and $12: 12 \mathrm{~h}$ photoperiod (D: L) [20]. 
Table 3. Matrix of experiments and evaporations rate (flux) $\mathrm{F}$ obtained for each test.

\begin{tabular}{lrrlll}
\hline Test & Factor 1 & Factor 2 & Factor 3 & Factor 4 & $\mathrm{~F}\left(\times 10^{4}\right)\left(\mathrm{g} \cdot \mathrm{h}^{-1} . \mathrm{cm}^{-1}\right)$ \\
\hline 1 & -1 & -1 & -1 & -1 & 1.2732 \\
2 & 1 & -1 & -1 & -1 & 3.8197 \\
3 & -1 & 1 & -1 & -1 & 2.5464 \\
4 & 1 & 1 & -1 & -1 & 11.4591 \\
5 & -1 & -1 & 1 & -1 & 1.2732 \\
6 & 1 & -1 & 1 & -1 & 1.2732 \\
7 & -1 & 1 & 1 & -1 & 2.5464 \\
8 & 1 & 1 & 1 & -1 & 2.5464 \\
9 & -1 & -1 & -1 & 1 & 0.6366 \\
10 & 1 & -1 & -1 & 1 & 0.6366 \\
11 & -1 & 1 & -1 & 1 & 2.2281 \\
12 & 1 & 1 & -1 & 1 & 2.5464 \\
13 & -1 & -1 & 1 & 1 & 1.2732 \\
14 & 1 & -1 & 1 & 1 & 1.2732 \\
15 & -1 & 1 & 1 & 1 & 2.8647 \\
16 & 1 & 1 & 1 & 1 & 3.5014 \\
\hline
\end{tabular}

$\mathrm{EOCa}$ or $\mathrm{EOCa}+\mathrm{RC}$ were placed in steel cylinders $(\mathrm{h}=0.5 \mathrm{~cm} ; \Phi=1 \mathrm{~cm}$ or $2 \mathrm{~cm}$ or $3 \mathrm{~cm})$, then the cylinders are included in glass petri dishes containing 10 insects. The assembly with a negative control (without product) are introduced into a fumigation chamber included in the experimental enclosure (temperature and relative humidity controlled). The mortality of insects is recorded as a function of time for $24 \mathrm{~h}$. Repetitions were performed in triplicate for each trial to minimize errors.

Corrected mortality in treating insects is expressed by equation (11):

$$
M \%=\left(\frac{M_{\text {test }}-M_{\text {control }}}{100-M_{\text {control }}}\right) \times 100
$$

with:

M\%: Mortality corrected;

$\mathrm{M}_{\text {test }}$ : Mortality observed during the test;

$\mathrm{M}_{\text {control }}$ : mortality observed in the control.

The determination of the lethal dose of $50 \%$ LD50 is determined by linear interpolation on curves giving the percentage of mortality as a function of the logarithm of the concentration tested.

\subsubsection{Optimization of transfer conditions}

In order to minimize the experimental conditions of diffusion transfer of EOCa essential oil in RC porous clay media, we performed a statistical approach based on experimental designs.

The transfer was presented as the evaporation rates $\mathrm{F}$ [21]. The design matrix (Full Factorial Design) was performed according to 4 factors $\mathrm{X}_{\mathrm{i}}$ such as:

- Factor $1=\mathrm{C}$ : Essential oil concentration $\left(0.01 \mu \mathrm{L} / \mathrm{cm}^{3}\right.$ and $\left.0.02 \mu \mathrm{L} / \mathrm{cm}^{3}\right)$;

- Factor $2=\mathrm{T}$ : Temperature $\left(25\right.$ and $\left.30^{\circ} \mathrm{C}\right)$;
- Factor 3=D: Cylinder diameter $(1 \mathrm{~cm}$ and $2 \mathrm{~cm})$; - Factor $4=\mathrm{M}$ : Mass of clay rock (0.05 g and $0.10 \mathrm{~g})$.

The number of tests carried out is calculated by the formula:

$$
\text { number of tests }=2^{k} \text {. }
$$

The number 2 corresponds to the number of two levels -1 and +1 and $\mathrm{k}$ stands for the number of factors studied.

In our study, the number of factors equals 4 :

number of tests $=2^{4}=16$

This corresponds to 16 tests.

Table 3 presents the matrix of the experiments and the evaporation rates obtained, from which the rows present the tests coded for the factors studied according to the high $(+1)$ or low $(-1)$ level of each factor.

The polynomial model presents:

$$
\begin{aligned}
F= & b_{0}+\sum_{i=1}^{n} b_{i} X_{i}+\sum_{i=1}^{n} \sum_{j=1}^{n-1} b_{i j} X_{i} X_{j} \\
& +\sum_{i=1}^{n} \sum_{j=1}^{n-1} \sum_{k=1}^{n-2} b_{i j k} X_{i} X_{j} X_{k}+b_{i j k l} X_{i} X_{j} X_{k} X_{l}
\end{aligned}
$$

Or:

- Mean: $b_{0}$;

-4 main effects: $b_{i}$;

- 6 effects present the 2 nd order interactions: $b_{i j}$;

-4 effects present the $3 r d$ order interactions: $b_{i j k}$;

-1 interaction of the 4 th order: $b_{i j k l}$.

2.3.3 Study of insecticidal activity behaviour of essential oil mixture in clay porous media

The interaction between the behaviour of EOCa in porous clay media RC and insecticidal activity was achieved by 
Table 4. $\mathrm{LD}_{50}$ of the insecticidal activities of the EOCa fixed on porous media.

\begin{tabular}{llll}
\hline Diameter $(\mathrm{cm})$ & Temperature $\left({ }^{\circ} \mathrm{C}\right)$ & $\mathrm{a}$ & $\mathrm{b}$ \\
\hline \multirow{2}{*}{$D_{1}=1$} & $T_{1}=25$ & 0.228 & 0.110 \\
& $T_{2}=30$ & 0.350 & 0.109 \\
& $T_{3}=35$ & 0.035 & 0.017 \\
$D_{2}=2$ & $T_{1}=25$ & 0.529 & 0.032 \\
& $T_{2}=30$ & 0.758 & 0.008 \\
& $T_{3}=35$ & 0.021 & 0.007 \\
$D_{3}=3$ & $T_{1}=25$ & $\gg$ & 0.056 \\
& $T_{2}=30$ & 0.061 & 0.017 \\
& $T_{3}=35$ & 0.013 & 0.005 \\
\hline
\end{tabular}

$\gg: \mathrm{LD}_{50}$ is greater than $1 \mu \mathrm{L} / \mathrm{cm}^{3}$. a: EOCa alone. b: EOCa $+\mathrm{RC}$ clay.

processing the matter transfer by diffusion parameters vs. mortality of insecticidal activity, according to the statistical tool of Principal Component Analysis (PCA) which is an extremely powerful tool for compressing and synthesizing information, useful when there is a sum of quantitative data to be processed, to be interpreted and to know the correlations between them [22].

\subsubsection{Computer and statistical processing}

All computer processing was done using MATLAB software. The numerical modelling results obtained will be compared with the experimental results, in order to find the parameters to be searched for and to make the maximum interpretation. Other computer tools have been used in parallel to perform certain calculations or graphical representation, such as: EXCEL, ORIGIN LAB, DESIGN PLAN and XLSTAT.

\section{Results and analysis}

\subsection{Insecticidal activities in porous clay media}

All the results of the insecticidal tests of the EOCa alone or with the porous clay media RC and GC, against Sitophilus granarius are displayed in Table 4 in the form of lethal doses of $50 \%\left(\mathrm{LD}_{50}\right)$ depending on the parameters studied such as: the diameter of cylinder D and the temperature of the incubation T. From this table, we deduce that the $\mathrm{LD}_{50}$ decrease in the presence of porous clayey $\mathrm{RC}$ and $\mathrm{GC}$ media, which proves that the insecticidal power has changed when the essential oil has been fixed on the porous media. In addition, the studied factors such as cylinder diameter and temperature keep the same properties of insecticidal activities, hence increasing cylinder diameter and increasing temperature promotes good activity. Also, it can be concluded that the insecticidal activity of the EOCa fixed on porous media and their persistence depends on the nature of the interactions between the constituent compounds of essential oils and the media studied [23].
Table 5. Parameters of the presented polynomial flux model for desorption.

\begin{tabular}{lrc}
\hline Coefficient & Value $^{(*)}$ & $p_{\text {test }}$ \\
\hline $\boldsymbol{b}_{0}$ & 2.6061 & 0.46 \\
$\boldsymbol{b}_{\boldsymbol{C}}$ & 0.7758 & 0.31 \\
$\boldsymbol{b}_{\boldsymbol{T}}$ & 1.1737 & 0.21 \\
$\boldsymbol{b}_{\boldsymbol{D}}$ & -0.5371 & 0.42 \\
$\boldsymbol{b}_{\boldsymbol{M}}$ & -0.7360 & 0.32 \\
$\boldsymbol{b}_{\mathrm{CT}}$ & 0.4575 & 0.47 \\
$\boldsymbol{b}_{\mathrm{CD}}$ & -0.6963 & 0.34 \\
$\boldsymbol{b}_{\mathrm{CM}}$ & -0.6565 & 0.36 \\
$\boldsymbol{b}_{\mathrm{TD}}$ & -0.3779 & 0.53 \\
$\boldsymbol{b}_{\mathrm{TM}}$ & -0.2586 & 0.64 \\
$\boldsymbol{b}_{\mathrm{DM}}$ & 0.8952 & 0.27 \\
$\boldsymbol{b}_{\mathrm{CTD}}$ & -0.3779 & 0.53 \\
$\boldsymbol{b}_{\mathrm{CTM}}$ & -0.3382 & 0.56 \\
$\boldsymbol{b}_{\mathrm{CD} M}$ & 0.7360 & 0.32 \\
$\boldsymbol{b}_{\mathrm{TDM}}$ & 0.4177 & 0.50 \\
$\boldsymbol{b}_{\mathrm{CTDM}}$ & 0.2745 & - \\
\hline
\end{tabular}

${ }^{*}$ The values presented are of the order of $\times 10^{4}\left(\mathrm{~g} \cdot \mathrm{h}^{-1} \cdot \mathrm{cm}^{-1}\right)$.

\subsection{Optimization of essential oil transfer conditions in porous media}

As we described previously, the experimental design chosen for the optimization of the flux parameter of the evaporation of the EOCa fixed in the porous medium is a full factorial design of four (4) factors: the concentration of 1 essential oil C, temperature T, cylinder diameter D and mass of clay medium M. Each factor requires 2 levels: high level is coded +1 and low level is coded -1 , which implies a total number of 16 simulations. Therefore, the mathematical model associated with the 1st degree polynomial is presented as a function of the factors plus the second, third and fourth order interactions of these factors.

The coefficients of the flux model of the essential oil fixed on the RC medium are displayed in Table 5. Following the results obtained by the optimization route, the mean flux is $2.6061 .10^{-4}$. If we take into account only the factors studied on the medium, we deduce that:

- The increasing concentration of the essential oil increases the flux;

- The increase in temperature increases the flow;

- The increase in cylinder diameter decreases the flow;

- The growth of the mass of porous medium decreases the flux.

The set of graphical representations of flux as a function of two factors in 3D are shown in Figure 2. The interaction of two of the four factors also influences the flux values. The flux increases with respect to the interaction of two concentration-temperature parameters, it decreases with respect to a parameter with the interactions of concentration-diameter, concentration-mass, mass-temperature and 


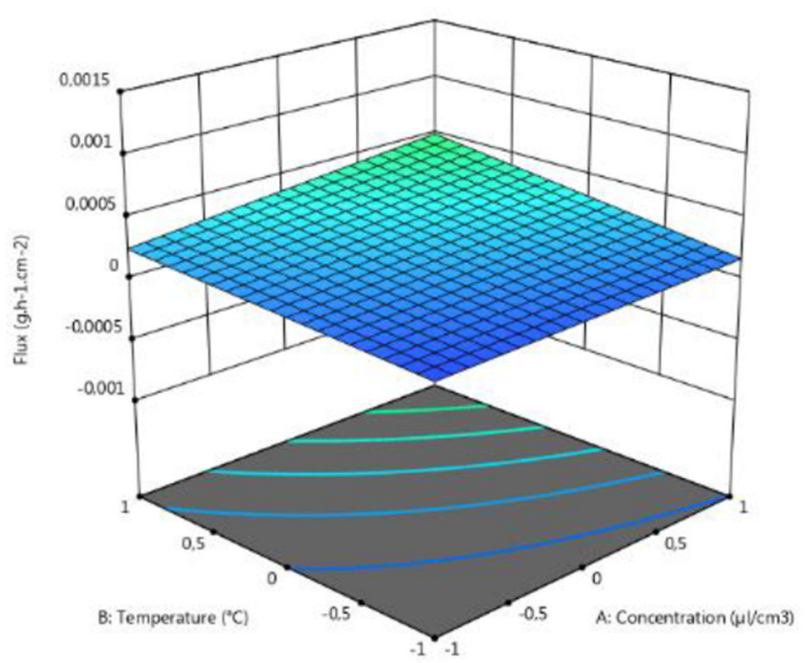

Température vs concentration

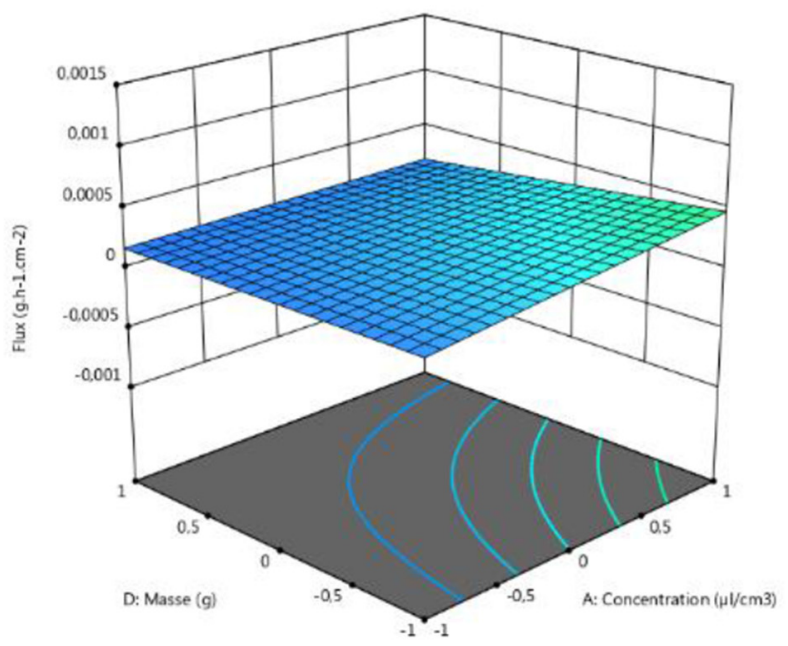

Masse vs concentration

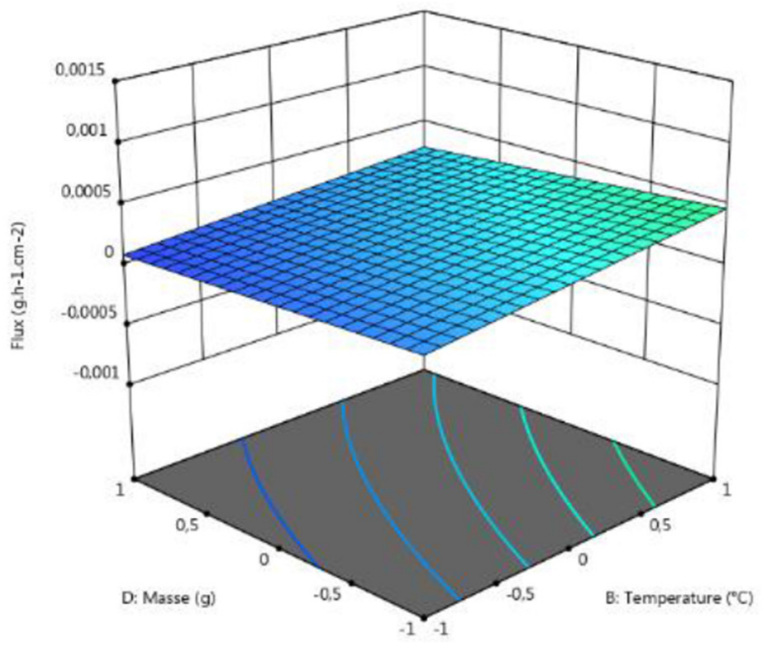

Masse vs température

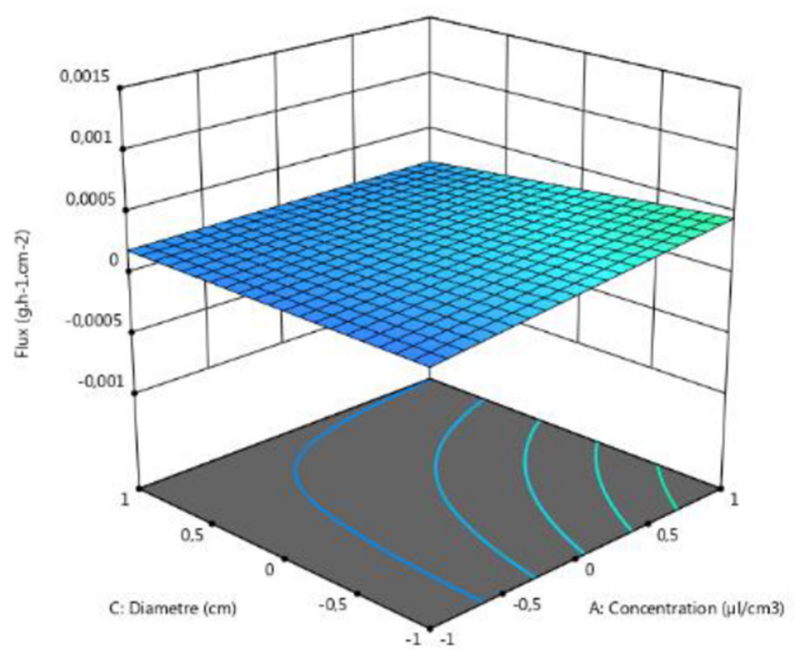

Diamètre vs concentration

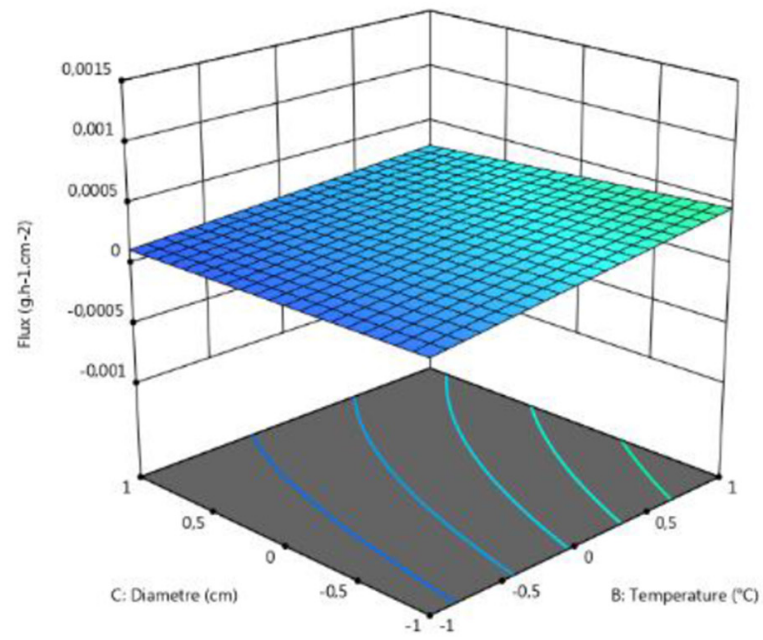

Diamètre vs température

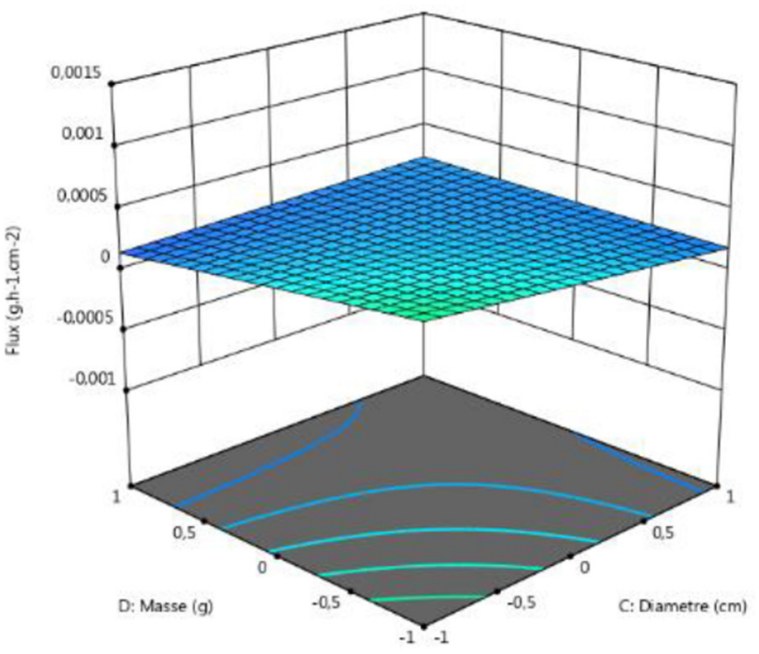

Masse vs diamètre

Fig. 2. 3D representation of flow as a function of two factors. 
Table 6. Statistical study of the presented polynomial model of flux during desorption.

\begin{tabular}{llllll}
\hline & dll & SSD & MSE & $\mathrm{F}_{\text {test }}$ & $\mathrm{p}_{\text {test }}$ \\
\hline Flux & 14 & $9.47 .10^{-7}$ & $6.76 .10^{-8}$ & 2.42 & 0.46 \\
$\boldsymbol{b}_{0}$ & 1 & $9.63 .10^{-8}$ & $9.63 .10^{-8}$ & 3.45 & 0.31 \\
$\boldsymbol{b}_{\boldsymbol{C}}$ & 1 & $2.20 .10^{-7}$ & $2.20 .10^{-7}$ & 7.89 & 0.21 \\
$\boldsymbol{b}_{\boldsymbol{T}}$ & 1 & $4.61 .10^{-8}$ & $4.61 .10^{-8}$ & 1.65 & 0.42 \\
$\boldsymbol{b}_{\boldsymbol{D}}$ & 1 & $8.66 .10^{-8}$ & $8.66 .10^{-8}$ & 3.10 & 0.32 \\
$\boldsymbol{b}_{\boldsymbol{M}}$ & 1 & $3.35 .10^{-8}$ & $3.35 .10^{-8}$ & 1.20 & 0.47 \\
$\boldsymbol{b}_{\mathrm{CT}}$ & 1 & $7.75 .10^{-8}$ & $7.75 .10^{-8}$ & 2.78 & 0.34 \\
$\boldsymbol{b}_{\mathrm{CD}}$ & 1 & $6.89 .10^{-8}$ & $6.89 .10^{-8}$ & 2.47 & 0.36 \\
$\boldsymbol{b}_{\mathrm{CM}}$ & 1 & $2.28 .10^{-8}$ & $2.28 .10^{-8}$ & 0.81 & 0.53 \\
$\boldsymbol{b}_{\mathrm{TD}}$ & 1 & $1.07 .10^{-8}$ & $1.07 .10^{-8}$ & 0.38 & 0.64 \\
$\boldsymbol{b}_{\mathrm{TM}}$ & 1 & $1.28 .10^{-7}$ & $1.28 .10^{-7}$ & 4.59 & 0.27 \\
$\boldsymbol{b}_{\text {DM }}$ & 1 & $2.28 .10^{-8}$ & $2.28 .10^{-8}$ & 0.81 & 0.53 \\
$\boldsymbol{b}_{\mathrm{CTD}}$ & 1 & $1.83 .10^{-8}$ & $1.83 .10^{-8}$ & 0.65 & 0.56 \\
$\boldsymbol{b}_{\mathrm{CTM}}$ & 1 & $8.66 .10^{-8}$ & $8.66 .10^{-8}$ & 3.10 & 0.32 \\
$\boldsymbol{b}_{\mathrm{CDM}}$ & 1 & $2.79 .10^{-8}$ & $2.79 .10^{-8}$ & 1.00 & 0.50 \\
Residue & 1 & $2.79 .10^{-8}$ & $2.79 .10^{-8}$ & & \\
Total & 15 & $9.75 .10^{-7}$ & & & \\
\hline
\end{tabular}

SSD: Sum of the squares of the deviations from the mean; MSE: Mean squared error=variance.

Table 7. Descriptive and predictive qualities of polynomial models.

\begin{tabular}{ll}
\hline Paramètre & Value \\
\hline Mean $^{*}$ & 2.60 \\
SD & 1.67 \\
Coefficient of variation $\boldsymbol{C} \boldsymbol{V}(\%)$ & 64.12 \\
Coefficient of determination $\boldsymbol{R}^{2}$ & 0.97 \\
Adjusted coefficient of determination $\boldsymbol{R}_{\boldsymbol{A}}^{2}$ & 0.57 \\
Predictive coefficient of determination $\boldsymbol{R}_{\text {pred }}^{2}$ & -6.33 \\
Response variation ratio & 6.68 \\
\hline
\end{tabular}

${ }^{*}$ The values presented are of the order of $\times 10^{4}\left(\mathrm{~g} \cdot \mathrm{h}^{-1} \cdot \mathrm{cm}^{-1}\right)$.

temperature-diameter and it decreases with respect to the interaction from two parameters to diameter-mass interactions. All the graphical representations of flow as a function of two factors in 3D are shown in Figure 2.

In general, and according to the results of Tables 6 and 7 , the coefficients of determination $\mathrm{R}^{2}$ for the two media show the correct explanation of the optimized models. The Fisher test value is $\mathrm{F}_{\text {test }}=2.42$ which explains why the model is not significant with respect to noise (uncertainties). The predictive coefficient of determination $\mathrm{R}_{\text {pred }}^{2}$ gives a negative value which implies that the total mean of flux presented can be a better predictor of the response of the model studied [24]. The variation ratio of the response measures the flux/uncertainty ratio, is equal to 6.68 which confirms that the response is adequate (ratio greater than 4 which is desirable).

\subsection{Modelling of the essential oil diffusion process in porous media}

To fully understand the behavior and the diffusion process of EOCa in the RC medium, two intermediate paths were made: the study of the desorption kinetics and the simulation of the concentration profiles inside the cylinder. This study makes it possible to explain the mechanism involved, as well as a means of precisely determining the parameters: the diffusion coefficient $\mathrm{D}_{\mathrm{z}}$, the evaporation flux $\mathrm{F}$, the evaporation constant $\mathrm{K}$ and the activation energy $\mathrm{E}_{\mathrm{a}}$.

Several kinetic models cited in the literature can study the desorption mechanism [25-27], in our case the model tested is the analytical solution of the Fickian diffusion model, which is described previously. The methodology adopted consists in controlling the mass percentage of the oil as a function of the time of the experimental results with simulations of analytical treatments during desorption. To make the simulation according to this model, it is necessary to seek the coefficient of diffusion $\mathrm{D}$ which is the principal parameter. The kinetic study was carried out under the optimal conditions corresponding to the tests carried out in the previous paragraph $\left(\mathrm{C}=0.01 \mu \mathrm{L} / \mathrm{cm}^{3}, \mathrm{~T}=30^{\circ} \mathrm{C}\right.$, $\mathrm{M}=0.10 \mathrm{~g}$ and $\mathrm{D}=2 \mathrm{~cm})$. The study of the linearity of equation (6) of the experimental results obtained by the gravimetric technique, gives us approximate values of the diffusion coefficients $\mathrm{D}_{\mathrm{z}}$ according to the optimal conditions, it is worth $9.81 .10^{-6} \mathrm{~cm}^{2} / \mathrm{h}$. The experimental results of the simulation of the desorption kinetics of the EOCa in the RC medium are shown in Figure 3. The curves obtained show a very clear branch which reflects a decrease 


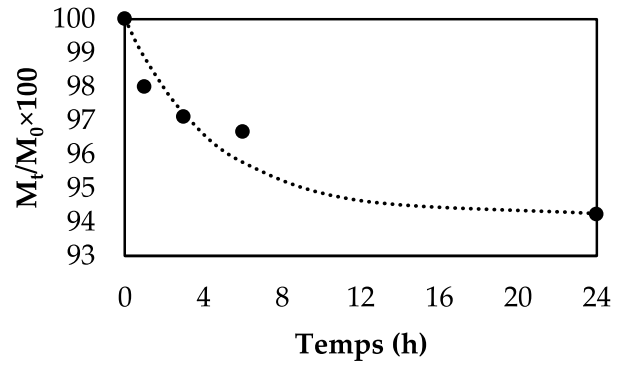

Fig. 3. Analytical simulation of the desorption kinetics of EOCa in the RC medium. (•): Experimental results; (...): Simulation, $C=0.01 \mu \mathrm{L} / \mathrm{cm}^{3} ; T=30{ }^{\circ} \mathrm{C} ; M=0.10 \mathrm{~g} ; D=2 \mathrm{~cm}$.

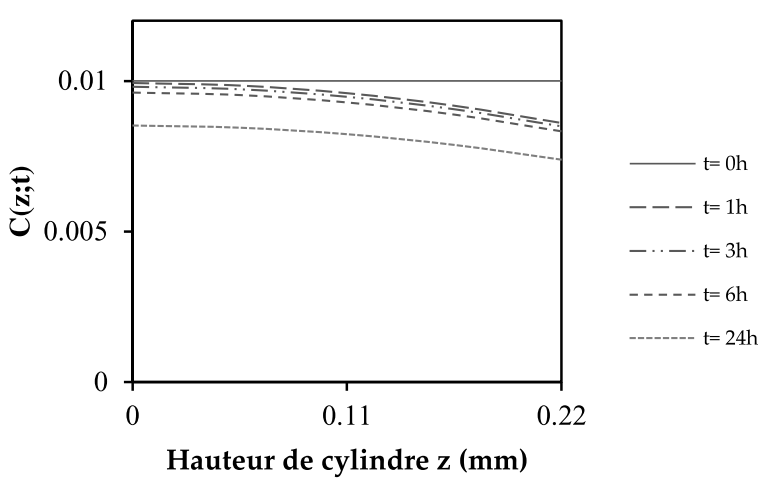

Fig. 4. Simulation of concentration profiles - Case of desorption of the EOCa in the RC medium.

in the mass percentage of the essential oil, which explains the desorption mechanism, we see that the amount desorbed is $5.77 \%$ over $24 \mathrm{~h}$.

The simulation made of the model is closer to the experimental results, which proves the validity of the analytical model developed and on the other hand the ability to give a reliable estimate of the other parameters to be sought. In general, the simulation has important interests, it can deduce the quantities of oil that remained during the time of biological applications (insecticidal activities). Figure 4 shows the simulation of the profiles of the concentration of essential oil in the medium. These concentration profiles are used to give good information about the transfer of EOCa within porous media, so that the concentrations can be determined for each altitude and for a more precise time.

\subsection{Interaction of the behavior of essential oils in porous media and insecticidal power}

As we described previously, the behavior of the EOCa in the $\mathrm{RC}$ medium is identified on the one hand by the diffusion parameters: the evaporation flux $\mathrm{F}$, the diffusivity $D_{z}$, the evaporation constant $K$ and the activation energy $\mathrm{E}_{\mathrm{a}}$, and insecticidal activity is presented by $\mathrm{M} \%$ mortality. The calculated values of all parameters are mentioned in Table 8, the interpretation of these results remains complex. On the other hand, the values of the energy of the activation are constant and low, which confirms that the two clay porous media have the same energetic behavior, moreover, the negative value proves the opposite direction of desorption (adsorption) and it shows the great capacity of porous media to fix essential oils [28].

Principal Component Analysis (PCA) is a statistical tool to study the behaviour of EOCa in RC media with respect to insecticidal activity and to determine hidden information. The case that we are going to study crosses 16 subjects (the matrices produced during the study of the optimization of the flow of evaporation in the two porous media) and the four (4) variables: the flow of evaporation $\mathrm{F}$, diffusivity $\mathrm{D}_{\mathrm{z}}$, evaporation constant $\mathrm{K}$ and mortality $\mathrm{M} \%$ (Tab. 8).

To fully explain the results, we must start with the choice of the axes that are interesting for our analysis. Table 9 present the eigenvalues of the four (4) presentative axes obtained. According to the Kaiser criterion [29], the axes $\mathrm{F} 1$ and $\mathrm{F} 2$ were retained whose eigenvalues are greater than $1 \quad\left(\lambda_{1}=1.89\right.$ and $\left.\lambda_{2}=1.38\right)$, with variabilities of $47.31 \%$ and $34.70 \%$ respectively, and corresponding to the total of $82 \%$ of the information. These eigenvalues of the axes selected return a "good proportion" of the analysis, this means that the sum of the inertia explained by each of the axes represents a significant part of the total inertia.

In the mapping displayed in Figure 5, we can see the following interpretations:

The parameters: the diffusion coefficient $D_{z}$ and the evaporation constant $\mathrm{K}$ are very close to the correlation circle towards the F1 axis and therefore very well represented on the mapping. The rather closed angle (starting from the origin) formed by the points $\left(D_{\mathrm{z}}: \cos ^{2}(\theta)=0.78\right.$ and $\left.\mathrm{K}: \cos ^{2}(\theta)=0.72\right)$, indicates that these two variables are well correlated with each other. These results come back to the dependence of these two parameters according to equation (9);

The parameters: the flow of evaporation $\mathrm{F}$ and the percentage of mortality $\mathrm{M} \%$ are close to the circle of correlation towards the axis F2, so they are well represented on the mapping. The rather closed angle formed by the points $\left(\mathrm{F}: \cos ^{2}(\theta)=0.24\right.$ and $\mathrm{M} \%$ : $\left.\cos ^{2}(\theta)=0.15\right)$, indicates that these two variables are well correlated between them. These results show that the observed M\% mortality of insecticide activity depends on the flow of evaporation $\mathrm{F}$;

On the other hand, the almost right angle formed by the four parameters indicates that the two variables of the diffusion coefficient $\mathrm{D}_{\mathrm{z}}$ and of the evaporation constant $\mathrm{K}$ are independent of the two other variables which are the evaporation flux $\mathrm{F}$ and the percentage mortality $\mathrm{M} \%$.

\section{General discussion}

The essential oil of Cedrus atlantica presented a good insecticidal activity against the stored commodity pest of wheat Sitophilus granarius, moreover, this activity was evolved when it was fixed in the clay porous medium. The mechanism of action of this activity is fumigation, hence the appropriate model of the process is a transfer of the material. 
Table 8. Parameters of diffusion and of insecticidal activity of EOCa desorption in the RC.

\begin{tabular}{|c|c|c|c|c|c|}
\hline Test & $\mathrm{F}\left(\times 10^{4}\right)\left(\mathrm{g} \cdot \mathrm{h}^{-1} \cdot \mathrm{cm}^{-1}\right)$ & $\mathrm{D}_{\mathrm{z}}\left(\times 10^{6}\right)\left(\mathrm{cm}^{2} \cdot \mathrm{h}^{-1}\right)$ & $\mathrm{K}\left(\times 10^{3}\right)$ & $\mathrm{E}_{\mathrm{a}}\left(\mathrm{J} \cdot \mathrm{mol}^{-1}\right)$ & $\mathrm{M} \%$ \\
\hline 1 & 1.2732 & 7.35 & 1.5 & \multirow{16}{*}{-8.176} & 16.66 \\
\hline 2 & 3.8197 & 1.47 & 0.3 & & 20 \\
\hline 3 & 2.5464 & 3.43 & 0.7 & & 20 \\
\hline 4 & 11.4591 & 2.45 & 0.5 & & 30 \\
\hline 5 & 1.2732 & 9.31 & 1.9 & & 20 \\
\hline 6 & 1.2732 & 2.94 & 0.6 & & 30 \\
\hline 7 & 2.5464 & 3.92 & 0.8 & & 23.33 \\
\hline 8 & 2.5464 & 1.96 & 0.4 & & 33.33 \\
\hline 9 & 0.6366 & 15.68 & 0.8 & & 20 \\
\hline 10 & 0.6366 & 5.88 & 0.3 & & 16.66 \\
\hline 11 & 2.2281 & 43.12 & 2.2 & & 23.33 \\
\hline 12 & 2.5464 & 17.64 & 0.9 & & 23.33 \\
\hline 13 & 1.2732 & 25.48 & 1.3 & & 20 \\
\hline 14 & 1.2732 & 9.8 & 0.5 & & 30 \\
\hline 15 & 2.8647 & 39.2 & 2.0 & & 30 \\
\hline 16 & 3.5014 & 21.56 & 1.1 & & 30 \\
\hline
\end{tabular}

Table 9. Eigenvalues of the presentative axes of the PCA.

\begin{tabular}{lllll}
\hline & F1 & F2 & F3 & F4 \\
\hline Eigenvalue & 1.89 & 1.38 & 0.53 & 0.18 \\
Variability (\%) & 47.31 & 34.70 & 13.45 & 4.53 \\
Cumulative (\%) & 47.31 & 82.00 & 95.46 & 100 \\
\hline
\end{tabular}

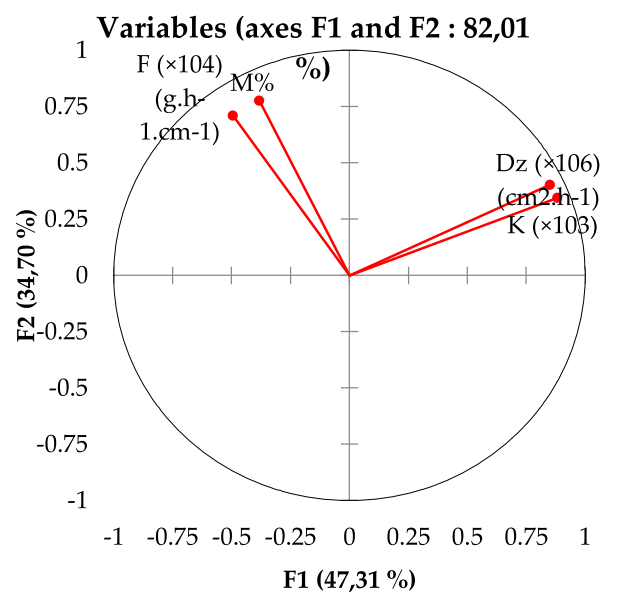

Fig. 5. Mapping of correlations between parameters of EOCa desorption and insecticidal activity.

To explain the mechanism, we have carried out a study of the relationship between the behavior of the essential oil in the porous medium with the insecticidal activity, hence the study of transfer of essential oil was done by using mathematical analytic models of Fickian diffusion processes under assumptions and theoretical approximations that facilitate numerical calculations. As a result, some physical parameters: the flux $\mathrm{F}$, the diffusivity $\mathrm{D}_{\mathrm{z}}$, the evaporation constant $\mathrm{K}$ were determined in parallel with the biological parameter of a mortality M\% of insecticidal activities against Sitophilus granarius. The use of the statistical tool of the principal component analysis (PCA) which consists in the determination of the correlation between all the obtained parameters allows to show that the biological activity is dependent on the flux F.

In parallel, the optimization of the diffusion transfer of the essential oil was done according to the design of experiments method with a Full factorial design. The results show that the flux $\mathrm{F}$ increases with the concentration of the essential oil and with the temperature, but decreases with the cylinder diameter (contact surface) and the mass of the porous medium.

In general, to have a good insecticidal activity against Sitophilus granarius it is necessary to play on the direct increase of the flow of the essential oil fixed on the porous medium by the increase of the concentration of the essential oil and the temperature and by the decrease of the cylinder diameter and the mass of the porous medium.

Finally, the limitations of this study can be cited as follows:

- The constituents of essential oils do not have the same affinity with respect to the porous medium;

- The insecticidal activity of the essential oil fixed in the porous medium and their persistence depends on the nature of interactions between the constituent compounds of the essential oil and the medium studied.

\section{Conclusion}

The study of the desorption behavior of Cedrus atlantica essential oil fixed on porous clay medium in relation to its insecticidal activities against Sitophilus granarius was 
carried out according to mathematical studies of Fick's law and well-defined statistical approaches such as principal component analysis and design of experiments. Several data and information were exploited to explain the mechanism of observation of the remarkable biological activity in the case of oil fixation in the porous medium.

The observed mortality of this insecticidal activity depends on the evaporation flow, and this last parameter also depends on the concentration of essential oil, the temperature, the diameter of the cylinder used and the mass of the porous medium. All the results of the data collected explain that the surface of the porous medium delays the affinity of the constituents of the oil (bioactive compounds) during the desorption.

\section{References}

1. S.A. Mir, B.N. Dar, M.A. Shah, S.A., Sofi, A.M. Hamdani, C.A. Oliveira, A.S. Sant'Ana, Application of new technologies in decontamination of mycotoxins in cereal grains: challenges, and perspectives, Food Chem. Toxicol. 111976 (2021)

2. Â. Luís, F. Domingues, A. Ramos, Production of hydrophobic zein-based films bioinspired by the lotus leaf surface: characterization and bioactive properties, Microorganisms 7, 267 (2019)

3. R.V. George, H.O. Harsh, P. Ray, A.K. Babu, Food quality traceability prototype for restaurants using blockchain and food quality data index, J. Clean. Prod. 240, 118021 (2019)

4. R.P. Guiné, S.G. Florença, M.J. Barroca, O. Anjos, The link between the consumer and the innovations in food product development, Foods 9, 1317 (2020)

5. S. Perera, S. Nanayakkara, M.N.N. Rodrigo, S. Senaratne, R. Weinand, Blockchain technology: Is it hype or real in the construction industry? J. Ind. Inf. Integr. 17, 100125 (2020)

6. J. Bauchet, S. Prieto, J. Ricker-Gilbert, Improved drying and storage practices that reduce aflatoxins in stored maize: experimental evidence from smallholders in Senegal, Am. J. Agric. Econ. 103, 296-316 (2021)

7. W.R. Morrison III, A. Lanba, B. Hall, A. Bruce, Novel implementation of laser ablation tomography as an alternative technique to assess grain quality and internal insect development in stored products, J. Stored Prod. Res. 86, $101552(2020)$

8. T. Vendl, M. Frankova, R. Aulicky, V. Stejskal, First record of the development of Sitophilus oryzae on two rodent bait formulations and literature overview of stored product arthropods infestations in rodent baits, J. Stored Prod. Res. 86, 101557 (2020)

9. J. Rösner, B. Wellmeyer, H. Merzendorfer, Tribolium castaneum: a model for investigating the mode of action of insecticides and mechanisms of resistance, Curr. Pharm. Des. 26, 3554-3568 (2020)

10. A.A. Warra, M.N.V. Prasad, African perspective of chemical usage in agriculture and horticulture-their impact on human health and environment, in: Agrochemicals Detection, Treatment and Remediation. Butterworth-Heinemann, 2020, pp. $401-436$
11. H. Musarurwa, N.T. Tavengwa, Emerging green solvents and their applications during pesticide analysis in food and environmental samples, Talanta 121507 (2020)

12. P. Pavez, R. Figueroa, M. Medina, D. Millán, R.D. Falcone, R.A. Tapia, Choline [amino acid] ionic liquid/water mixtures: a triple effect for the degradation of an organophosphorus pesticide, ACS Omega 5, 26562-26572 (2020)

13. M.B. Isman, Commercial development of plant essential oils and their constituents as active ingredients in bioinsecticides, Phytochem. Rev. 19, 235-241 (2020)

14. N. Aoussar, N. Rhallabi, R.A. Mhand, R. Manzali, M. Bouksaim, A. Douira, F. Mellouki, Seasonal variation of antioxidant activity and phenolic content of Pseudevernia furfuracea, Evernia prunastri and Ramalina farinacea from Morocco, J. Saudi Soc. Agric. Sci. 19, 1-6 (2020)

15. S. Zrira, M. Ghanmi, Chemical composition and antibacterial activity of the essential of Cedrus atlantica (Cedarwood oil), J. Essent. Oil Bear. Plants 19, 1267-1272 (2016)

16. A. Ainane, M. Taleb, F. El-Hajjaji, B. Hammouti, A. Chetouani, T. Ainane, dependence between two types of most abundant natural clays in Bejaad province (Central Morocco) using a statistical approach, Mor. J. Chem. 9, 210-220 (2021)

17. A. Ainane, F. Khammour, S. Charaf, M. Elabboubi, M. Elkouali, M. Talbi, T. Ainane, Chemical composition and insecticidal activity of five essential oils: Cedrus atlantica, Citrus limonum, Rosmarinus officinalis, Syzygium aromaticum and Eucalyptus globules, Mater. Today Proc. 13, 474-485 (2019)

18. J. Crank, The mathematics of diffusion (Oxford University Press, 1979), pp. 105-254

19. M.B. Teixeira, S. Tobinaga, A diffusion model for describing water transport in round squid mantle during drying with a moisture-dependent effective diffusivity, J. Food Eng. 36, 169-181 (1998)

20. A. Ainane, F. Khammour, M. Elkouali, M. Talbi, A. Oussaid, A. Lemhidi, T. Ainane, Evaluation of the toxicity of the essential oils of certain mints grown in the region of Settat (Morocco): Mentha piperita, Mentha pulegium and Mentha spicata against, Sitophilus Granarius, Sitophilus Oryzae and Sitophilus Zeamais, J. Anal. Sci. Appl. Biotechnol. 1, 1-10 (2019)

21. L.Á. Oliveira, J.C. Santos, T.H. Panzera, R.T. Freire, L.M. Vieira, F. Scarpa, Evaluation of hybrid-short-coir-fibrereinforced composites via full factorial design, Composite Structures 202, 313-323 (2018)

22. D. Granato, J.S. Santos, G.B. Escher, B.L. Ferreira, R.M. Maggio, Use of principal component analysis (PCA) and hierarchical cluster analysis (HCA) for multivariate association between bioactive compounds and functional properties in foods: A critical perspective, Trends Food Sci. Technol. 72, 83-90 (2018)

23. S. Gharby, A. Asdadi, M. Ibourki, A. Hamdouch, T. Ainane, L.A.I. Hassani, Chemical characterization of the essential oil from aerial parts of Lavandula rejdalii Upson \& Jury, a medicinal plant endemic to Morocco, J. Essent. Oil Bear. Plants 23, 1422-1427 (2020)

24. F. Yang, C. Long, Z. Wei, L. Long, Optimization of medium using response surface methodology to enhance the growth of Effrenium voratum (Symbiodiniaceae, Dinophyceae), J. Phycol. 56, 1208-1215 (2020) 
25. A. Tursi, F. Chidichimo, R. Bagetta, A. Beneduci, BTX removal from open aqueous systems by modified cellulose fibers and evaluation of competitive evaporation kinetics, Water 12, 3154 (2020)

26. G.C. Leandro, C. Capello, B.L. Koop, J. Garcez, A.R. Monteiro, G.A. Valencia, Adsorption-desorption of anthocyanins from jambolan (Syzygium cumini) fruit in laponite ${ }^{\circledR}$ platelets: Kinetic models, physicochemical characterization, and functional properties of biohybrids, Food Res. Int. 109903 (2020)
27. F.A. Houle, R.E. Miles, C.J. Pollak, J.P. Reid, A purely kinetic description of the evaporation of water droplets, J. Chem. Phys. 154, 054501 (2021)

28. J. Foroozesh, S. Kumar, Nanoparticles behaviors in porous media: application to enhanced oil recovery, J. Mol. Liq. 113876 (2020)

29. S. Asante-Okyere, C. Shen, Y.Y. Ziggah, M.M. Rulegeya, $\mathrm{X}$. Zhu, Principal component analysis (PCA) based hybrid models for the accurate estimation of reservoir water saturation, Comput. Geosci. 145, 104555 (2020)

Cite this article as: Ayoub Ainane, Fatouma Mohamed Abdoul-Latif, Jalludin Mohamed, Wissal Attahar, Manar Ouassil, Zine Laabidine Shybat, Adnane El Yaacoubi, Tarik Ainane, Behaviour desorption study of the essential oil of Cedrus atlantica in a porous clay versus insecticidal activity against Sitophilus granarius: explanation of the phenomenon by statistical studies, Int. J. Metrol. Qual. Eng. 12, 12 (2021) 